\title{
Densidad Asimétrica Neuronal Inter-Hemisférica de la Corteza Insular en Ratones FMR1 Knock-Out
}

\author{
Interhemispheric Neural Density Asymmetry of Insular Cortex in FMR1 Knock-Out Mice
}

\author{
N. Ramírez*; C. Maestu*; M. Ramos* \& F. del Pozo*
}

RAMÍREZ, N.; MAESTU, C.; RAMOS, M. \& DEL POZO, F. Densidad asimétrica neuronal inter-hemisférica de la corteza insular en ratones FMR1 Knock-Out. Int. J. Morphol., 32(4):1377-1382, 2014.

RESUMEN: El síndrome X frágil en los seres humanos es causado por una mutación en el gen FMR1 y se asocia con grave retraso mental, hiperactividad y ansiedad. Hemos comparado ratones FMR1-KO con ratones Control en la densidad neuronal de la corteza insular, área del cerebro asociada con el procesamiento del dolor y manejo de la ansiedad. Los ratones también fueron sometidos a una prueba de aprendizaje espacial en un entorno de ansiedad. Los resultados muestran asimetría significativa entre la densidad neuronal entre ínsula izquierda y derecha en $\mathrm{KO}$ en comparación con ratones de tipo Control. En cuanto al comportamiento, a pesar de los ratones KO no mostraron marcados déficits en la realización de tareas mostraron una velocidad superior a la de sus homólogos de tipo Control. Por otra parte, la asimetría de densidad insular se correlaciona con una mayor velocidad a nivel individual. Estos resultados sugieren que la asimetría de la densidad neuronal insular en FMR1 ratones KO se puede considerar como un correlato anatómico de las anormalidades de comportamiento observados.

PALABRAS CLAVE: FMRP; FMR1; Corteza insular; Dolor; Ansiedad; Modelo animal.

\section{INTRODUCCIÓN}

El síndrome $X$ frágil es una patología neurodegenerativa, es la forma más común de discapacidad intelectual hereditaria. El X frágil no tiene cura. La causa genética es un tipo de mutación conocido como expansión de repeticiones de trinucleótidos, que afecta a una región del cromosoma X en la que se sitúa el gen FMR-1. La expansión del trinucleótido tiene lugar en la región reguladora del gen, siendo este trinucleótido CGG (Citosina-GuaninaGuanina). Cuando el número de repeticiones supera el valor umbral de 230 repeticiones se produce la metilación del gen y, por tanto, éste pierde su función, produciendo así el síndrome del X frágil. Las enfermedades producidas por mutaciones en regiones no codificadoras son típicamente trastornos multisistémicos que involucran la disfunción o degeneración de diferentes tejidos. En los individuos de la población general, el número de repeticiones CGG es polimórfico y se sitúa entre 6 y 50. Las repeticiones en este rango son estables cuando se transmiten de generación a generación. En cambio, cuando el número de CGGs supera las 200 (mutación completa), el gen FMR1 se silencia dando lugar a la ausencia de FMRP y a las manifestaciones clínicas del SXF: RM y rasgos característicos. Finalmente, los individuos con alelos de entre 55 y
200 repeticiones CGG son los denominados portadores de la premutación. En esta situación, el gen se transcribe, hay síntesis de FMRP y por tanto, los individuos permutados son asintomáticos para SXF, no presenta RM. A pesar de ello, presentan un riesgo incrementado a tener descendencia afectada ya que el número de repeticiones es inestable y tiende a incrementar en cada división celular. Estas dos alteraciones son: la insuficiencia ovárica primaria asociada al síndrome $\mathrm{X}$ frágil (FXPOI), que causa una menopausia antes de los 40 años, y un síndrome de ataxia y temblor conocido como FXTAS (fragileX-associated tremor/ataxia syndrome).

Ya que la proteína FMR1 es importante para la formación y desarrollo del cerebro, los signos y síntomas que se presentan en el síndrome X frágil están: retraso mental, fenotipo típico, con defectos del tejido conectivo (ligamentos) el cual es laxo o flexible, el comportamiento puede ser muy sociable hasta retraído o agresivo. Los estímulos sensoriales como ruidos, contacto físico y presencia de muchas otras personas pueden agobiar a las personas con FXS (Hagerman \& Hagerman, 2002; Loesch \& Hagerman, 2012; Loomis et al., 2012; McLennan et al., 2011). 
Los cambios patológicos en el cerebro de pacientes FXS se observan en los estudios de resonancia magnética, incluyen anormalidades en el vermis (Reiss et al., 1991) del cerebelo y posterior, disminución del volumen de la circunvolución temporal superior (Reiss et al., 1994), y un volumen caudado ampliada (Reiss et al., 1995). Protuberancias dendríticas anormales también se han descrito en esta población (Segal et al., 2000). Se cree que estas dos anormalidades estructurales del cerebro y el fenotipo observado en estructural FXS son causados por la ausencia de la proteína FMRP. En Ratones adultos FMR1 KO se ha demostrado que tienen espinas dendríticas anormalmente largas (Castrén et al., 2005; Bureau et al., 2008). Además, que se correlaciona con el comportamiento patológico en este tipo de ratones (Restivo et al., 2005; Peier et al., 2000). La Densidad neuronal es una medida importante histológica. Por ejemplo, el estrés crónico se ha asociado con cambios en la densidad neuronal en ratones de tipo Control (Olivares et al., 2004, 2010).

La ínsula es un área de especial interés en relación con FXS debido a su papel en el procesamiento del dolor y manejo de la ansiedad. Muchas áreas del cerebro están involucradas en la experiencia del dolor. Técnicas de mapeo cerebral en los seres humanos y los animales han aportado muchos conocimientos sobre las regiones del cerebro que inervan el procesamiento del dolor. En particular, la corteza insular que sirve como interfaz entre los sistemas de procesamiento aferentes y las áreas responsables de la orientación cognitiva (Starr et al., 2009; Papathanasiou et al., 2006; Craig, 2003). Existe evidencia a partir de modelos animales de FXS de los déficits en la amplificación de las señales de dolor entrantes en el nivel espinal (Price \& Melemedjian, 2012). Una alta proporción de portadores humanos femeninos de una premutación en el gen FMR1 sufren dolor musculoesquelético generalizado. También hay un mayor riesgo de ser diagnosticados con fibromialgia, una condición asociada con los umbrales de dolor disminuido y déficits cognitivos, además de dolor musculo esquelético, especialmente en la presencia de signos motores del síndrome de temblor asociado ataxia X-frágil (FXTAS) (Leehey et al., 2011). Otro síntoma importante asociado con FXS es la ansiedad. La ínsula parece estar implicado en la representación de experiencias adversivas como el miedo y la ansiedad (Bagni et al., 2012; Paulus \& Stein, 2006).

\section{MATERIAL Y MÉTODO}

Modelo Animal. Ratones Mus musculus ratones C57BL / 6 FMR1 KO fueron adquiridos del Fondo para Erasmus Animal / Erasmus Dierexperimenteel Centrum (EDC) (http:// www.erasmusmc.nl/edc/). Estos animales están destinados para su uso en la investigación biológica única. Todos los procedimientos fueron aprobados por el Comité de Ética institucional de la Universidad Complutense de Madrid y se llevaron a cabo de acuerdo con el artículo 4 de la Directiva 92/65 CEE del Consejo en relación con los requisitos de sanidad animal. 9 hembras y 9 machos (13 a 16 meses de edad) de tipo Control (WT) y, 9 hembras y 9 machos (13-16 meses de edad) ratones FMR1 KO. Los ratones se mantuvieron con $12 \mathrm{~h}$ de luz, $12 \mathrm{~h}$ oscuras. Todas las pruebas de comportamiento se llevaron a cabo durante la fase de luz, 07:00 a.m. - 07:00 p.m.

Genotipo FMRP. El Genotipado de FMR1 se realizó con PCR, la biopsia con cola de ratón y el protocolo utilizado fue el Jackson Laboratory (Bar Harbor, Maine, EE.UU., http://jaxmice.jax.org/). El emparejamiento fue estrictamente controlado. Además, de que cada sujeto descendiente (ratón) fue Genotipado.

Prueba espacial de memoria. Se empleó el laberinto Barnes (Barnes, 1979) para caracterizar el aprendizaje espacial y la memoria, este permite medir la capacidad de un ratón para aprender y recordar la ubicación de una zona mediante una configuración de señales visuales distales situados alrededor de la zona de pruebas. Las tareas utilizadas son útiles para identificar los déficits cognitivos en cepas transgénicas de roedores que el modelo para la enfermedad tales como FXS (Harrison et al., 2006, 2009). El laberinto Barnes consiste en una superficie circular con 20 orificios circulares colocados equidistantes cerca de la orilla de la mesa. Las indicaciones visuales se colocan alrededor de la mesa a la vista del animal. Bajo uno de los agujeros hay una "caja de escape", que podría ser alcanzada por el roedor a través del orificio correspondiente en la superficie de la mesa. Los estímulos adversivas son luz $(150 \mathrm{w})$ y sonido $(80 \mathrm{~dB})$ obligándolo a encontrar refugio. Los parámetros medidos una vez que el ratón es liberado hasta que se encuentra la caja de escape son: velocidad media y distancia recorrida.

El laberinto de Barnes se divide en 4 fases. Antes de todas las fases del laberinto la mesa debe estar completamente limpia eliminando todas las fuentes de olor para evitar señales olfativas.

Fase 1: Adaptación. El ratón se coloca en el centro del laberinto. No hay elementos de adversivas. Después de tres minutos, el ratón se induce suavemente para entrar en la caja de escape, si no lo ha hecho antes. Esto se repite una segunda vez después de un intervalo de 15 min.

Fase 2: adquisición espacial. Luz adversivas (150 w) y sonido (80 dB) estimulación se introducen en esta etapa. Los estímulos adversivos son puestos una vez que el ratón es 


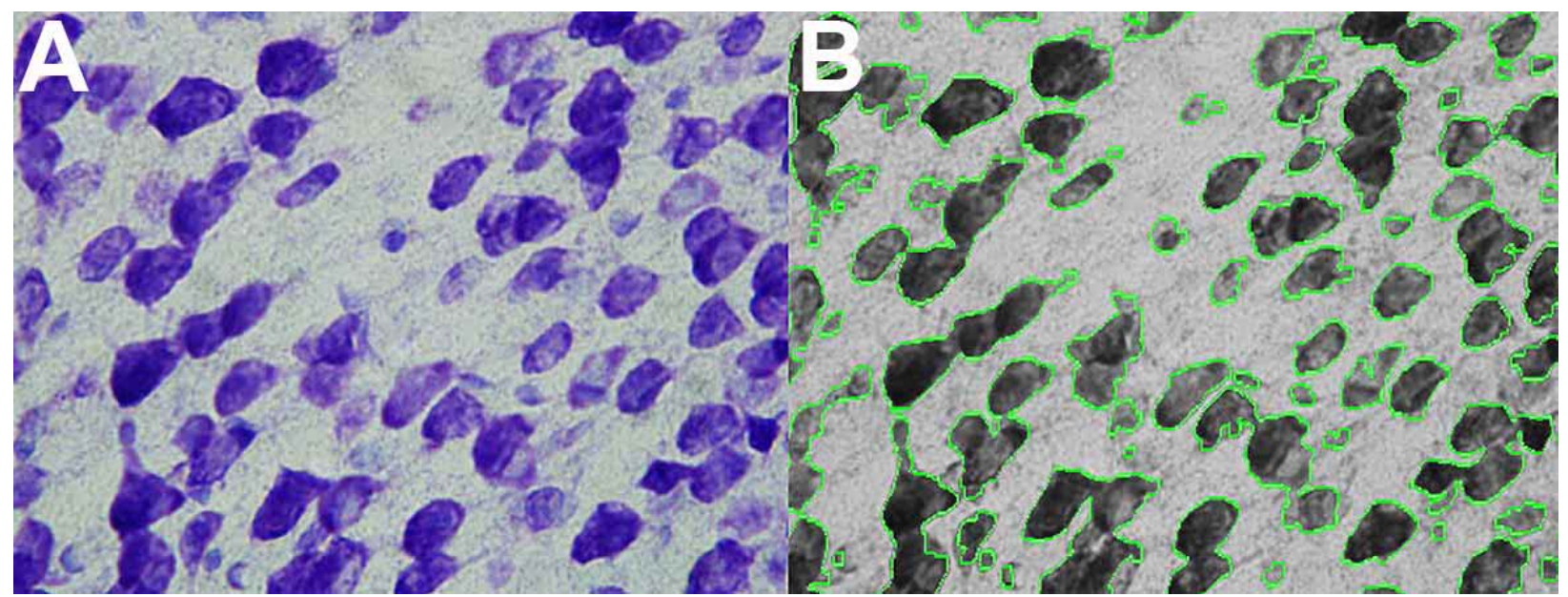

Fig 1. A). Imagen original a color. B) Imagen en Intensidad con la detección de bordes y estimación densidad.

puesto en el centro del laberinto y se retiran cuando el ratón entra en la caja de escape. Al igual que antes, el ratón se induce suavemente para entrar en la caja de escape, si no lo ha hecho después de tres minutos. Cuatro sesiones por día, con un intervalo entre sesiones se llevaron a cabo. Esto se repitió durante 4 días consecutivos.

Fase 3: Memoria de Corto plazo. El día 5, 24 h después de la última jornada de entrenamiento, se suelta el ratón en el centro del laberinto y es libre de encontrar la caja de escape, mientras que los estímulos aversivos se entregan. La sesión se termina después de $90 \mathrm{seg}$. Si el ratón no ha llegado a la caja de salida por ese tiempo que se induce suavemente para entrar en la caja y los estímulos adversivos se desconectan.

Fase 4: A largo plazo tarea de memoria. Esta fase tenía la misma estructura que el anterior con la diferencia de que se llevó a cabo en 12 d.

Preparación del corte cerebral. El animal fue anestesiado con ketamina y luego se sacrificó por perfusión de fijador (paraformaldehído al 4\%) en la aorta. Un breve período de perfusión salina precedió al fijador. Después de que el cerebro se fijó, se extrajo del cráneo y queda sumergida en sacarosa durante 7 días a una temperatura de $4^{\circ} \mathrm{C}$ para completar el proceso de fijación. Los cortes fueron de tipo coronal a 50 micras de espesor con el equipo automático Vibratome. Los cortes sin procesar en crioprotector sacarosa y luego se congelaron a $-20^{\circ} \mathrm{C}$. Se realizó un corte en el lado derecho de identificar los dos hemisferios.

Procesamiento de la imagen de Histología. Se analizaron un total de 383 de cortes coronales para estimar la densidad neuronal (190 desde la izquierda y 193 del hemisferio derecho) a partir de los 36 ratones descritos anteriormente. Un mínimo de 5 cortes se obtuvieron de cada ratón y hemisferio. Las imágenes digitales fueron tomadas con un microscopio electrónico Olympus BX51. Para evaluar la reproducibilidad dos análisis diferentes se llevaron a cabo en dos escalas diferentes (40X y 100X). Ambos análisis arrojaron resultados equivalentes. Desarrollamos una aplicación bajo la plataforma de MatLab (Mathworks, Natwick, California, EE.UU., Fig. 1) para el análisis automático de la imagen. Los resultados fueron validados manualmente con aplicaciones similares comerciales tal como MetaMorph (Microscopía Automatización y Análisis de Imágenes Software es el estándar de la industria para la adquisición de microscopio automatizado) y ImageJ (Procesamiento de Imágenes y Análisis en Java, http://imagej.nih.gov/ij/). Como un primer paso, las imágenes en color se convierten a escala de grises. A continuación, el contraste se mejoró utilizando adaptativa ecualización del histograma contraste limitado. Las imágenes resultantes fueron convertidas a blanco y negro. Los píxeles colindantes se combinaron a continuación, utilizando la interpolación bilineal para eliminar límites inducidas artificialmente. A partir de estas imágenes se detectaron fronteras y superficies cerradas llenas de color negro. Estas imágenes finales permiten el cálculo de la densidad neuronal como un porcentaje.

\section{RESULTADOS}

Prueba espacial de memoria. Las variables independientes consideradas fueron genotipo (KO y WT) y el sexo (masculino y femenino). Como se describe en la sección Métodos, las variables dependientes medidas fueron el tiempo transcurrido de la prueba hasta que encuentra la caja de escape (duración), velocidad media y distancia recorrida. El análisis se llevó a cabo de forma independiente para las fa- 

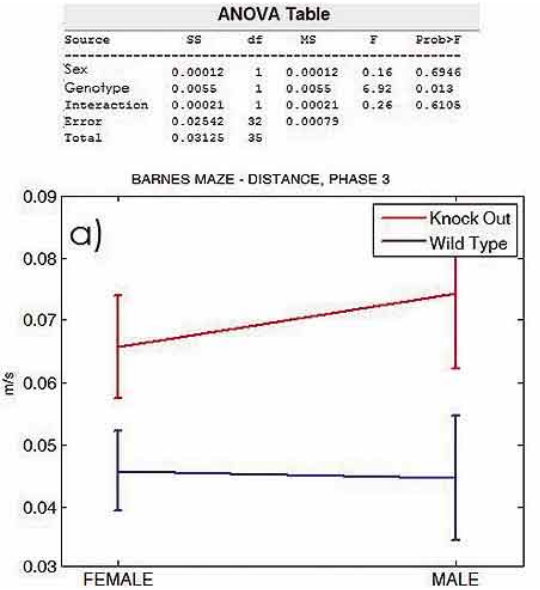
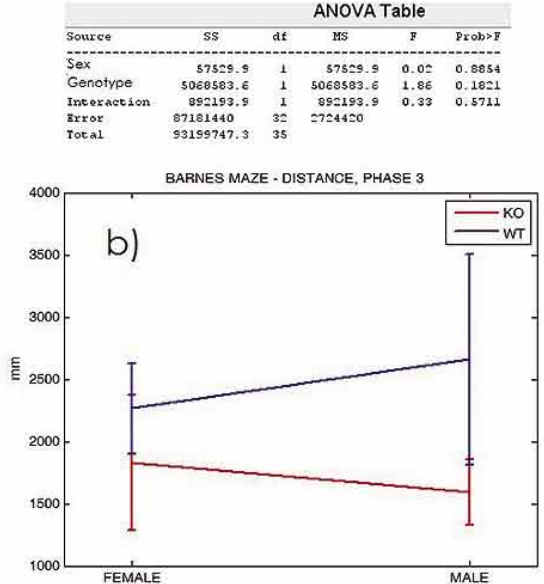
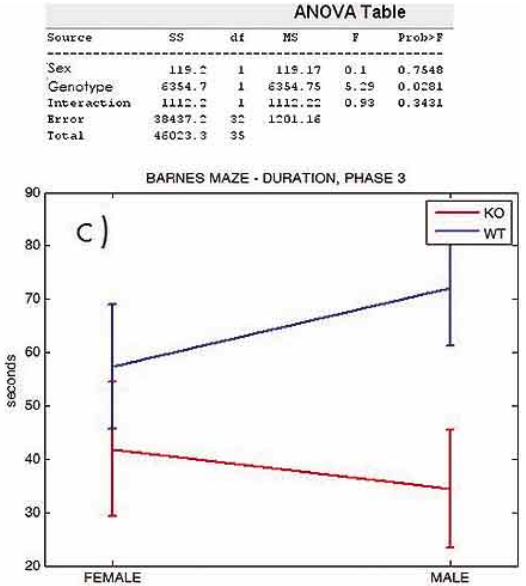

Fig. 2. El análisis estadístico (ANOVA) muestra que los ratones knock-out eran más rápidos y tomaron menos tiempo en el laberinto de la tarea de memoria a corto plazo. No se encontraron diferencias con respecto a la distancia recorrida. A) Velocidad, B) Distancia recorrida, C) Duración.

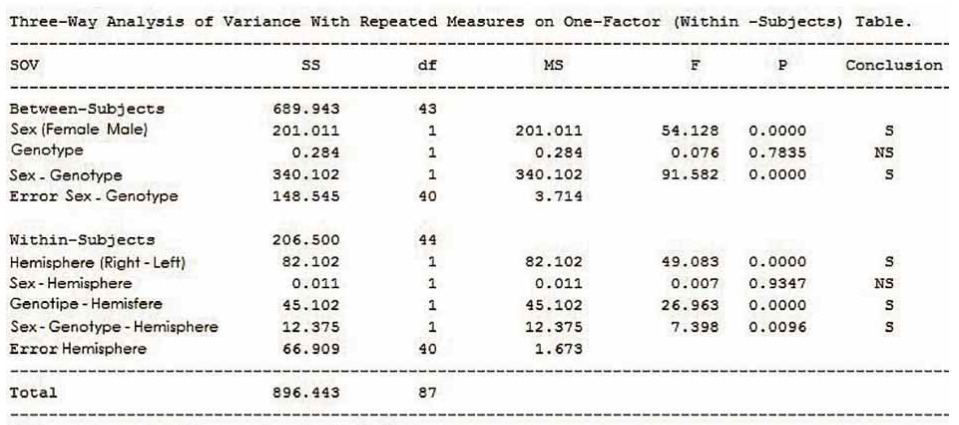

With a given significance level of: 0.05

The results are significant (S) or not significant (NS).
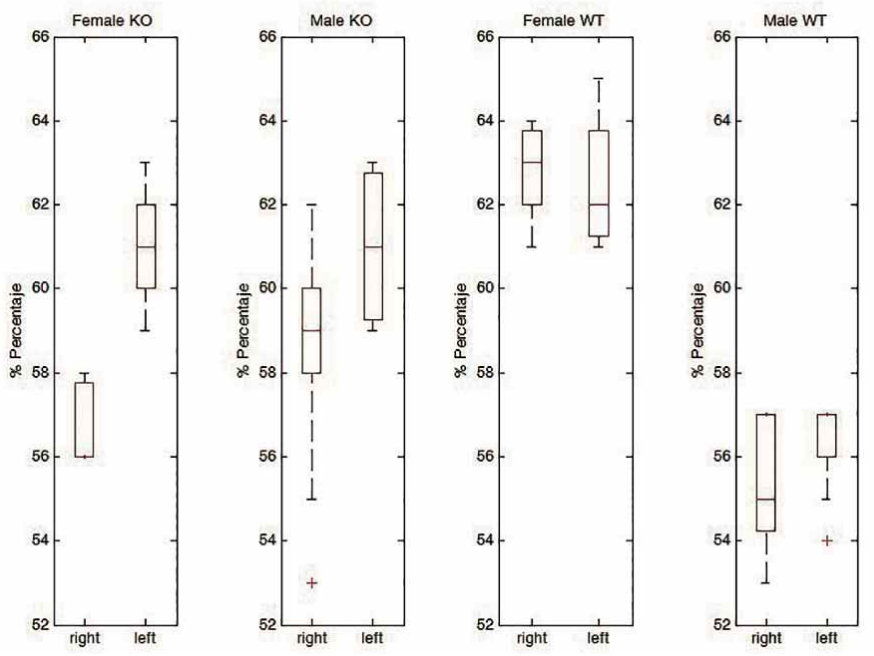

Fig. 3. Resultados del diseño mixto ANOVA de 3 vías de la densidad neuronal insular con el sexo y el genotipo como dentro de los factores de sujeto y de hemisferio como en factor de tema.

ses 3 (memoria a corto plazo) y 4 (memoria a largo plazo). Un análisis de 2 vías de la varianza (ANOVA, Fig. 2) mostró que los ratones KO eran significativamente más rápido y tomó menos tiempo que sus homólogos WT. No se encontraron diferencias significativas con respecto al sexo o la distancia recorrida. Se encontraron los mismos resultados tanto a corto plazo y la memoria a largo plazo (datos no mostrados).

Densidad Neuronal. En primer lugar, se realizó un triple análisis- diseño mixto de la varianza con la densidad neuronal insular como variable dependiente, el sexo (femenino y masculino) y el genotipo (KO, WT) como entre los sujetos variables independientes y hemisferio (izquierda y derecha) como sujeto dentro de la variable independiente. Distribución de la densidad neuronal por categoría se muestra en la Figura 3. La tabla de ANOVA muestra que todas las variables independientes mostraron diferencias significativas excepto para el genotipo. Del mismo modo, todas las interacciones fueron significativas, excepto para el hemisferio X sexo (Fig. 3). A continuación realizó post-hoc de dos vías ANOVAs para cada genotipo por separado con el sexo como factor de entre sujeto y el hemisferio como en factor de tema. Los resultados indican diferencias significativas con respecto al hemisferio para los ratones KO (Fig. 4A) con una mayor densidad neuronal en el hemisferio izquierdo. Para los animales WT las diferencias significativas fueron con respecto al sexo, con los ratones hembra que muestra la densidad neuronal mayor insular (Fig. 4B).

Resultados de Correlación Histológica y Prueba de Comportamiento. Para investigar las posibles relaciones entre los datos histológicos y de comportamiento, se calculó el coeficiente de co- 

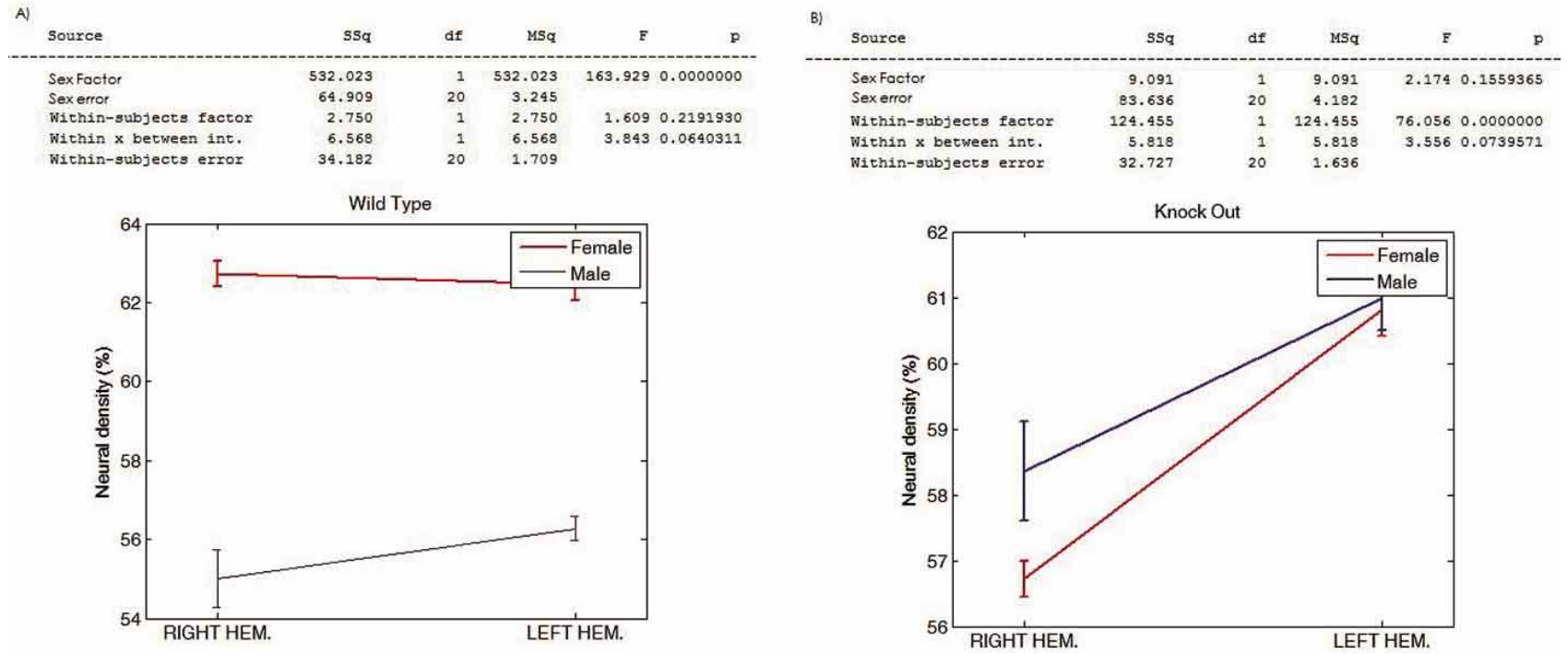

Fig. 4. Post-hoc de 2 vías ANOVAs para cada genotipo muestran diferencias significativas con respecto al hemisferio para los ratones KO y con respecto al sexo de los animales WT.

rrelación de Pearson entre la asimetría de densidad hemisferio a nivel individual definidos como Asimetría $=2 *$ (Densidad Derecha - Densidad Izquierda) / (densidad derecha + Densidad Izquierda) y los diversos conductual se incluyeron velocidad, duración y distancia recorrida, tanto para la fase 3 y 4 ratones correspondientes a ambos sexos y genotipos. Se aplicó una corrección de Bonferroni para comparaciones múltiples. Se encontró correlación significativa entre la fase 4 la velocidad y la asimetría ( $r=-0,48$, Pcorregida $<0,05$, Fig. 5).

\section{DISCUSIÓN}

El presente estudio compara la densidad neuronal de la ínsula, y medidas de comportamiento que refleja el aprendizaje espacial de ratones FMR1 KO y WT. Los resultados histológicos más relevantes fueron que los ratones KO mostraron una asimetría densidad neuronal interhemisférica mientras que esto no era el caso de los animales WT. En cuanto al comportamiento, los ratones $\mathrm{KO}$ con los estímulos adversivos mostraron mayor velocidad y menor tiempo para completar la tarea que los WT. Además, se encontró una cantidad significativa de correlación a nivel de ratón individual entre la asimetría interhemisférica y la tarea de velocidad en memoria a largo plazo (fase 4).

La relación entre el SXF-corteza insular y SXF-ansiedad, se ha explorado en trabajos anteriores. El Volumen Insular se ha encontrado reducido en los seres humanos con SXF en comparación con controles sanos usando MRI

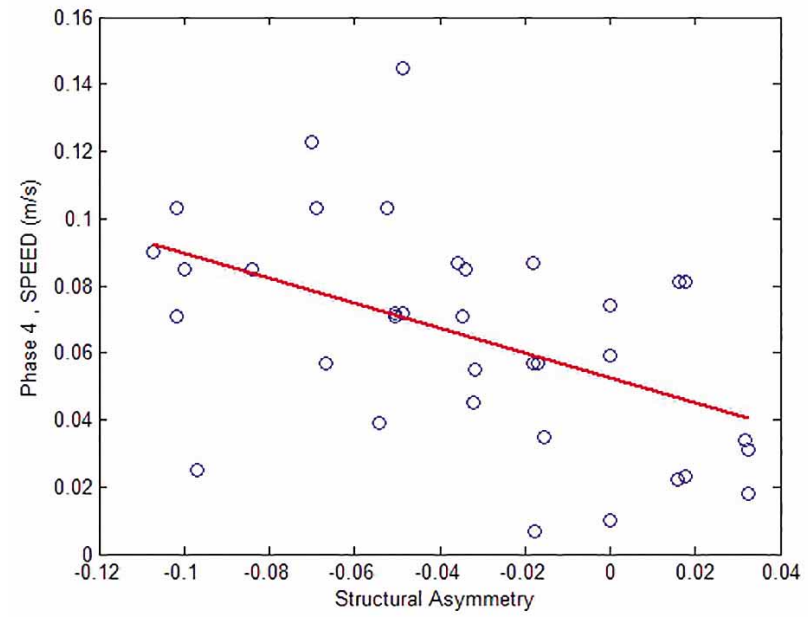

Fig. 5. Diagrama de dispersión de asimetría interhemisférica densidad neuronal vs velocidad para la Fase 4. La línea roja es el resultado de una regresión lineal.

(Cohen et al., 2011). Los pacientes con SXF normalmente sufren de ansiedad (Bagni et al.). Del mismo modo, los ratones FMR1 KO se han visto alterados la ansiedad y el comportamiento social y, por tanto, constituir un modelo animal es relevante para la ansiedad en el comportamiento por parte de los pacientes con SXF. Además, se cree que la ínsula a desempeñar un papel clave en los procesos que median el nivel de ansiedad.

Los resultados del presente estudio sugieren que las asimetrías insulares descritas pueden constituir un correlato anatómico de algunas de las anomalías de comportamiento presentes en los ratones transgénicos, que a su vez, pueden tener implicaciones para la relación entre la morfología insular y el SXF en los seres humanos. 
RAMÍreZ, N.; MAESTU, C.; RAMOS, M. \& DEL POZO, F. Interhemispheric neural density asymmetry of insular cortex in FMR1 Knock-out mice. Int. J. Morphol., 32(4):1377-1382, 2014.

SUMMARY: Fragile $\mathrm{X}$ syndrome in humans is caused by a mutation in the FMR1 gene and it is associated with severe mental retardation, hyperactivity and anxiety. Here we compare FMR1 KnockOut mice, a model of Fragile-X syndrome, and wild-type mice with respect to the neuronal density of the insular cortex, a brain area associated with pain processing and anxiety management. Mice were also subjected to a spatial learning test in an anxiogenic environment. Results show significant asymmetry between neuronal density between left and right insula in knock out as compared to wild type mice. Behaviorally, although knock-out mice did not show deficits in task completion they explored the maze at a higher velocity than their wild-type counterparts. Furthermore, insular density asymmetry correlated with higher velocity during one of the spatial navigation tasks at the individual mouse level. These results suggest that insular neuronal density asymmetry in FMR1 Knot-Out mice may be considered as an anatomical correlate of the observed behavioral abnormalities

KEY WORDS: FMRP; FMR1; Insular cortex; Pain way; Anxiety; Animal model.

\section{REFERENCIAS BIBLIOGRÁFICAS}

Bagni, C.; Tassone, F.; Neri, G. \& Hagerman, R. Fragile X syndrome: causes, diagnosis, mechanisms, and therapeutics. J. Clin. Invest., 122(12):431422, 2012.

Barnes, C. A. Memory deficits associated with senescence: a neurophysiological and behavioral study in the rat. J. Comp. Physiol. Psychol., 93(1):74-104, 1979.

Bureau, I.; Shepherd, G. M. \& Svoboda, K. Circuit and plasticity defects in the developing somatosensory cortex of FMR1 knock-out mice. J. Neurosci., 28(20):5178-88, 2008

Castrén, M.; Tervonen, T.; Kärkkäinen, V.; Heinonen, S.; Castrén, E.; Larsson, K.; Bakker, C. E.; Oostra, B. A. \& Akerman, K. Altered differentiation of neural stem cells in fragile $\mathrm{X}$ syndrome. Proc. Natl. Acad. Sci. USA, 102(49):17834-9, 2005.

Cohen, J. D.; Nichols, T.; Brignone, L.; Hall, S. S. \& Reiss, A. L. Insular volume reduction in fragile X syndrome. Int. J. Dev. Neurosci., 29(4):489-94, 2011.

Craig, A. D. Pain mechanisms: labeled lines versus convergence in central processing. Annu. Rev. Neurosci., 26:1-30, 2003.

Hagerman, R. J. \& Hagerman, P. J. (Eds.). Fragile X Syndrome: Diagnosis, Treatment, and Research. 3rd ed. Baltimore, Johns Hopkins University Press, 2002

Harrison, F. E.; Hosseini, A. H. \& MacDonald, M. P. Endogenous anxiety and stress responses in water maze and Barnes maze spatial memory tasks. Behav. Brain Res., 198(1):247-51, 2009.

Harrison, F. E.; Reiserer, R. S.; Tomarken, A. J. \& McDonald, M. P. Spatial and nonspatial escape strategies in the Barnes maze. Learn. Mem., 13(6):80919, 2006.

Leehey, M. A.; Legg, W.; Tassone, F. \& Hagerman, R. Fibromyalgia in fragile X mental retardation 1 gene premutation carriers. Rheumatology (Oxford), 50(12):2233-6, 2011.
Loesch, D. \& Hagerman, R. Unstable mutations in the FMR1 gene and the phenotypes. Adv. Exp. Med. Biol., 769:78-114, 2012.

Loomis, E. W.; Eid, J. S.; Peluso, P.; Yin, J.; Hickey, L.; Rank, D.; McCalmon, S.; Hagerman, R. J.; Tassone, F. \& Hagerman, P. J. Sequencing the unsequenceable: expanded CGG-repeat alleles of the fragile $\mathrm{X}$ gene. Genome Res., 23(1):121-8, 2013.

McLennan, Y.; Polussa, J.; Tassone, F. \& Hagerman, R. Fragile x syndrome. Curr. Genomics, 12(3):216-24, 2011.

Olivares, R.; Toledo, C.; Vera, Y.; Morgan, C.; Pérez, H.; Ortíz, A.; Henríquez, M.; Hernández, A. \& Cifuentes, F. Density in primary visual cortex (17 visual area) from rats subjected to chronic stress. Int. J. Morphol., 28(3):85560, 2010 .

Olivares, R.; Godoy, G.; Adaro, L. \& Aboitiz, F. Neuronal density in primary visual cortex (17 visual area), in two wild rodent species. Int. J. Morphol., 22(4):279-84, 2004.

Papathanasiou, E. S.; Papacostas, S. S.; Charalambous, M.; Eracleous, E.; Thodi, C. \& Pantzaris, M. Vertigo and imbalance caused by a small lesion in the anterior insula. Electromyogr. Clin. Neurophysiol., 46(3):185-92, 2006.

Paulus, M. P. \& Stein, M. B. An insular view of anxiety. Biol. Psychiatry, 60(4):383-7, 2006.

Peier, A. M.; Mcllwain, K. L.; Kenneson, A.; Warren, S. T.; Paylor, R. \& Nelson, D. L. (Over)correction of FMR1 deficiency with YAC transgenics: behavioral and physical features. Hum. Mol. Genet., 9(8):1145-59, 2000.

Price, T. J. \& Melemedjian, O. K. Fragile X Mental Retardation Protein (FMRP) and the Spinal Sensory System. Results Probl. Cell Differ., 54:41-59, 2012.

Reiss, A. L.; Abrams, M. T.; Greenlaw, R.; Freund, L. \& Denckla, M. B. Neurodevelopmental effects of the FMR-1 full mutation in humans. Nat. Med., 1(2):159-67, 1995.

Reiss, A. L.; Freund, L.; Tseng, J. E. \& Joshi, P. K. Neuroanatomy in fragile X females: the posterior fossa. Am. J. Hum. Genet., 49(2):279-88, 1991.

Reiss, A. L.; Lee, J. \& Freund, L. Neuroanatomy of fragile X syndrome: the temporal lobe. Neurology, 44(7):1317-24, 1994.

Restivo, L.; Ferrari, F.; Passino, E.; Sgobio, C.; Bock, J.; Oostra, B. A.; Bagni, C. \& Ammassari-Teule, M. Enriched environment promotes behavioral and morphological recovery in a mouse model for the fragile $\mathrm{X}$ syndrome. Proc. Natl. Acad. Sci. USA, 102(32):11557-62, 2005.

Segal, I.; Korkotian, E. \& Murphy, D. D. Dendritic spine formation and pruning: common cellular mechanisms? Trends Neurosci., 23(2):53-7, 2000.

Starr, C. J.; Sawaki, L.; Wittenberg, G. F.; Burdette, J. H.; Oshiro, Y.; Quevedo, A. S. \& Coghill, R. C. Roles of the insular cortex in the modulation of pain: insights from brain lesions. J. Neurosci., 29(9):2684-94, 2009.

\section{Dirección para Correspondencia: \\ Ceferino Maestru}

Universidad Politécnica de Madrid (UPM) / Centro de Tecnología Biomédica (CTB),

Campus Montegancedo, 28223

Pozuelo de Alarcón

Madrid

ESPAÑA

Recibido : 12-08-2014

Aceptado: 22-09-2014

Email: ceferino.maestu@ctb.upm.es 